\title{
Amphiphilic copolymers for liquid bandage application studies
}

\section{Zhiqi Zhang ${ }^{1}$, Anthony Conway ${ }^{2}$, Ann Beal Salamone ${ }^{2}$, Eric T. Crumpler ${ }^{1}$, Xueji Zhang ${ }^{3,4}$, Chen-zhong Li ${ }^{1}$}

${ }^{I}$ Nanobioengineering/Bioelectronics Lab, Department of Biomedical Engineering, Florida International University, 10555 W. Flagler St., Miami, Florida 33174, USA, ${ }^{2}$ Rochal Industries LLP, 12719 Cranes Mill, San Antonio, TX 78230, USA, ${ }^{3}$ Institue of Biomedicine and Bioengineering, University of Science and Technology, Beijing, Beijing 100083, P.R. China, ${ }^{4}$ Department of Chemistry, University of South Florida, 4202 E. Fowler Ave., CHE 205A, Tampa, Florida 33620, USA

\section{TABLE OF CONOTENTS}

\author{
1. Abstract \\ 2. Introduction \\ 3. Material and methods \\ 3.1. Materials \\ 3.2. Polymer synthesis and polymer formulation preparation \\ 3.2.1. Polymer I (poly (TRIS-co-NIPAM)), formulation 1 \\ 3.2.2. Polymer II (Poly (TRIS-co-NIPAM-co-DFHA)), formulation 3 \\ 3.2.3. 3.2.3. Polymer III (Poly (TRIS-co-NVP)), formulation 4 \\ 3.2.4. Polymer Formulation 6 \\ 3.3. ${ }^{I} H$ NMR spectroscopy and molecular weight determination \\ 3.4. Polymer characterization \\ 3.5. Scanning electron microscopy (SEM) imaging \\ 3.6. Cell culture and sample plate preparation \\ 3.7. SRB cell viability assay \\ 3.8. Statistical analysis \\ 4. Results \\ 4.1. Cell viability SRB analysis \\ 4.2. Polymer characterizations \\ 5. Discussion \\ 5.1. Polymer characterizations \\ 5.2. Fixation method optimization \\ 6. Conclusions \\ 5.3. Cell viability \\ 7. Acknowledgement \\ 8. References
}

\section{ABSTRACT}

This research pertains to a new class of liquid bandage polymers which are promising for assisting advanced wound healing by serving as substrates to promote cell viability and proliferation. Amphiphilic nitrogen-containing polymer poly (3methacryloyloxypropyltris (trimethylsiloxy)silane-co-Nisopropylacrylamide) (poly (TRIS-co-NIPAM)) was synthesized and investigated with further comparison to several different wound care polymers including commercialized 3M Nexcare ${ }^{\mathrm{TM}}$ No Sting Liquid Bandage. Cell viability on different polymers was tested on fetal human skin fibroblasts (HSFs) and neonatal human epidermal keratinocytes (HEKs). Test results were quantified by Sulforhodamine B (SRB) in vitro cytotoxicity assay. It is demonstrated that both HSFs and HEKs survive better on the poly (TRIS-co-NIPAM) film as the cell seeding substrate compared to other candidate polymer formulations, as well as to the commercial $3 \mathrm{M}$ No Sting Liquid Bandage polymer. Thus we conclude that wound healing could be accelerated by this new class of liquid bandage polymer, particularly for early-stage wounds due to a cell substrating effect.

\section{INTRODUCTION}

Wound healing has been an on going research interest for dermatologists and tissue engineers. The primary purpose of the wounding healing products is to protect skin from degradation caused by infection, exposure to body fluids, incontinence, tape trauma and/or friction. As a class of topical skin treatment products, liquid bandages are increasingly used in hospitals, nursing homes and home health care settings. Liquid bandages are usually mixtures of chemicals which form a polymeric film on top of the skin. The preferred liquid bandage polymeric film should be air and water-vapor permeable which provides a conformable adherent to the covered area (1). Biocompatibility and low cytotoxicity are also generally considered as fundamental yet crucial characteristics in processes such as wound healing (1-3). An ideal liquid bandage should also have enhanced cell adhesion properties to promote cell growth in order to facilitate the wound healing process (4). However, none of the commercially available liquid bandages have been reported to support cell growth.

Thermally responsive NIPAM based hydrogels have been evaluated with promising potential as substrates 
for cell viability and tissue formation with bovine articular chondrocytes (3), human pancreatic tissue (5), rat primary hepatocytes and with bovine carotid endothelial cells $(6,7)$. Homopolymers of NIPAM have a lower critical solution temperature (LCST) between $32^{\circ} \mathrm{C}$ and $35^{\circ} \mathrm{C}$ in water, which makes this type of monomer an excellent candidate in polymeric form to release water and aggregate (by a reduction in polymer chain dimensions) when applied to the human skin or mucosal tissue $(7,8)$. It has been reported that the LCST of NIPAM copolymers with hydrophobic monomers leads to a decrease in the LCST of poly-NIPAM in water (approximately $32{ }^{\circ} \mathrm{C}$ ). Thus, if NIPAM is copolymerized with the oxygen and water-vapor permeable hydrophobic monomer TRIS, the reduction in polymer chain dimensions caused by aggregation of the NIPAM phases at body temperature could be expected to contribute to the void volume in the polymer film. Consequently, increasing oxygen and water vapor permeability are expected. This also provides enhanced cohesion of the polymer films and enhanced adhesion to a biological surface.

In our research, we investigated a new class of amphiphilic liquid bandage polymers forming a conformable and non-tacky film on skin surfaces, while maintaining sufficient oxygen and water-vapor permeability. Several different formulations of polymers were synthesized and tested for cell viability and proliferation. The unique chemistry of the liquid bandage polymers of this study were amphiphilic copolymers composed of the hydrophobic siloxy-containing monomer and hydrophilic nitrogen-containing monomers. The hydrophobic siloxy-containing monomer was trimethylsiloxy (TRIS), while the hydrophilic monomers studied were NIPAM and N-vinylpyrrolidone (NVP). Hexamethyldisiloxane (HMDS) was used as the solvent to facilitate fast drying and to have no stinging to the skin (9, 10). The polymers of this study appear to be capable of functioning as substrates on a wound surface and wound edges to support cell adhesion and cell migration for reepithelialization (11). Similar copolymers of hydrophobic TRIS with hydrophobic comonomers have been used for many years in medical devices, such as HMDS-containing liquid adhesive bandages as well as contact lenses, with no known incidence of cytotoxicity $(9,12)$. The ability for the polymers discussed in this paper to support cell adhesion and proliferation is considered, an important attribute for potentially improving wound healing.

In our study, cell viability and proliferation are demonstrated by the ability of cells to attach to the polymer film surfaces. Keratinocytes are the major cell type of the epidermis, making up more than $90 \%$ of the epidermal layer (10). Fibroblasts are connective tissue cells (dermis) that secrete an extracellular matrix rich in collagen and other macromolecules (11). Both cell types play critical roles in apical wound healing and often are used as indicators for testing biocompatibility and cell proliferation. In vitro cell-culture systems of neonatal epidermal keratinocytes (HEK) and fetal human skin fibroblasts (HSF) as mimetic elements of the skin matrix were employed in this assessment. Cell viability was determined by SRB colorimetric assay for the biocompatibility of the copolymers as substrates for these two types of cells.

\section{MATERIALS AND METHODS}

\subsection{Materials}

HMDS was obtained from Dow Corning (Midland, MI). 3-Methacryloyloxypropyltris (trimethylsiloxy)silane (TRIS) was obtained from Silar Laboratories (Scotia, NY). N-Isopropylacrylamide (NIPAM) was purchased from Jarchem Industries (Newark, $\mathrm{NJ}$ ). $\quad \mathrm{N}$-vinyl-2-pyrrolidone (NVP) and $1 \mathrm{H}, 1 \mathrm{H}, 7 \mathrm{H}-$ dodecafluoroheptyl methacrylate were purchased from Polysciences (Warrington, PA). N,N-Dimethylacrylamide was purchased from Alfa Aesar (Ward Hill, MA). 2, 2'Azobis (2-methylbutyronitrile) was obtained from either Wako Chemicals USA (Richmond, VA) or DuPont Chemical Solutions Enterprise (Wilmington, DE). Sucrose acetate isobutyrate was obtained from Eastman Chemical Company (Kingsport, TN). Reagent grade ethyl acetate was purchased from Puritan Products (Bethelem, PA) and reagent grade acetone was purchased from VWR International (West Chester, PA). NexCare ${ }^{\mathrm{TM}}$ Liquid Bandage and FC-43 ${ }^{\mathrm{TM}}$ (perfluorotributylamine) were obtained from 3M (St. Paul, MN). All chemicals were used as received without further purification with the exception of purification procedures used following polymerization of the monomers.

Fetal Human Skin Fibroblasts (HSFs) and modified Eagles Minimum Essential Medium (EMEM) were obtained from American Type Culture Collection (ATCC, Manassas, VA). Neonatal Epidermal Keratinocytes (HEKs) Total Kit and HEK Growth Medium were obtained from Cell Applications (San Diego, CA). Bottled distilled water, Phosphate Buffered Saline (PBS), Hank's Balanced Salt Solution (HBSS) and Fetal Bovine Serum (FBS) were purchased from Gibco Life (Grand Island, NY). Pipettes and pipette tips were purchased from Fisher Scientific. Other cell culture reagents including Human Lung Collagen Type I and $0.25 \%$ Trypsin-EDTA solution as well as SRB based in vitro Toxicology Assay Kit (including $0.4 \%$ SRB solution, trichloroacetic acid (TCA), acetic acid and $10 \mathrm{mM}$ unbuffered Trizma base solution), and additional $99.5 \%$ acetic acid were purchased from Sigma Aldrich (St. Louis, MO). The plate reader used was TECAN GNEios Multiplate Reader by Phenix Research (Candler, NC). Formal Fixx was purchased from Thermo Shandon (Pittsburgh, PA). 96-well flat bottom plates were purchased from Becton Dickinson Labware (Franklin Lakes, NJ).

\subsection{Polymer Synthesis and Polymer Formulation Preparation}

Three amphiphilic polymers varying in the hydrophilic monomers (NIPAM, VNP and DFHA) were synthesized. Five different formulations were produced from the copolymers by formulation modifications. The ratio of hydrophilic to hydrophobic monomers in the copolymer was adjusted to render the coating insoluble in water after the volatile, hydrophobic solvent had 


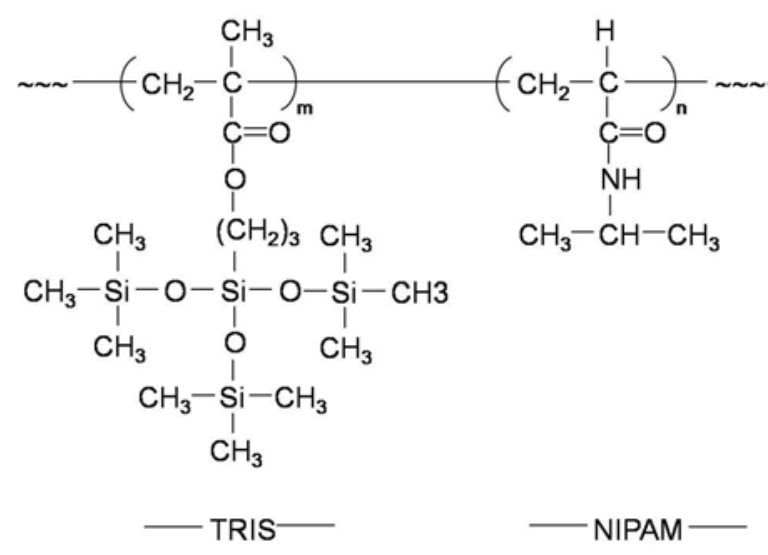

Figure 1. Poly(TRIS-co-NIPAM) synthesis.

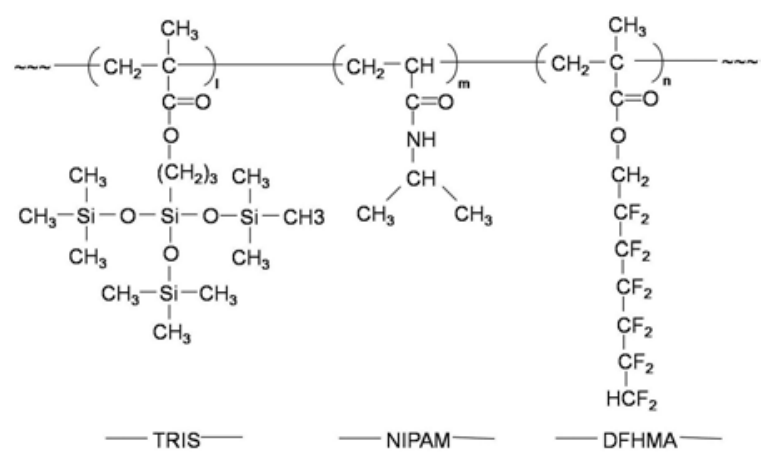

Figure 2. Poly(TRIS-co-NIPAM-co-DFHMA) synthesis.

evaporated. Synthesis methods of selected polymer formulations are described in the following text.

3.3. Polymer I (poly (TRIS-co-NIPAM)), formulation 1:

A $25 \mathrm{ml}$ reaction vessel was charged with $16.0 \mathrm{~g}$ ethyl acetate, $3 \mathrm{~g}(0.007 \mathrm{~mol})$ TRIS, $1 \mathrm{~g}(0.009 \mathrm{~mol})$ NIPAM and 0.08 g 2,2'-azobis (2-methylbutanenitrile). After nitrogen flushing for $3 \mathrm{~min}$, the vessel was closed and placed in an oil bath. The free radical polymerization was run for $17 \mathrm{hr}$ at $68-72{ }^{\circ} \mathrm{C}$. The polymer was precipitated into water, filtered, dissolved in acetone, reprecipitated into water, filtered and dried at $20{ }^{\circ} \mathrm{C}$. Following synthesis and purification, poly (TRIS-co-NIPAM) $(10 \mathrm{wt} \%)$ and sucrose acetate isobutyrate (2 wt \%) were dissolved in HMDS to produce Polymer Formulation 1. The product chemical structure is shown in Figure 1.

\subsection{Polymer II (Poly (TRIS-co-NIPAM-co-DFHA)), formulation 3: \\ A $25 \mathrm{ml}$ reaction vessel was charged with $15 \mathrm{~g}$} ethyl acetate, $3.23 \mathrm{~g}(0.008 \mathrm{~mol})$ TRIS, $1.09 \mathrm{~g}(0.010 \mathrm{~mol})$ NIPAM, 0.69 g (0.002 mol) 2,2,3,3,4,4,5,5,6,6,7,7dodecafluoroheptyl methacrylate (DFHA) and $0.101 \mathrm{~g} 2,2$ 'azobis (2-methylbutanenitrile). After nitrogen flushing for $3 \mathrm{~min}$, the vessel was closed and placed in an oil bath. The polymerization was run for $15.5 \mathrm{hr}$ at $63-79{ }^{\circ} \mathrm{C}$. The polymer was precipitated into water, filtered, dissolved in acetone, reprecipitated in water and dried at $50{ }^{\circ} \mathrm{C}$ resulting in an $83 \%$ yield. The polymer was compatible in HMDS at $10 \mathrm{wt} \%$ polymer in the liquid. Following synthesis and purification, poly (TRIS-co-NIPAM-co-DFHA) (10 wt \%) and FC-43 $(<1 \mathrm{wt} \%)$ were dissolved in HMDS to produce Polymer Formulation 3 . The product chemical structure is shown in Figure 2.

\subsection{Polymer III (Poly (TRIS-co-NVP)), formulation 4}

A $25 \mathrm{ml}$ reaction vessel was charged with $16 \mathrm{~g}$ ethyl acetate, $4.91 \mathrm{~g}(0.012 \mathrm{~mol})$ TRIS, $1.11 \mathrm{~g}(0.010 \mathrm{~mol})$ $\mathrm{N}$-vinylpyrrolidone (NVP) and 0.12 g. 2,2'-azobis (2methylbutanenitrile). After nitrogen flushing for $3 \mathrm{~min}$, the vessel was closed and placed in an oil bath. The free radical polymerization was run for $15.5 \mathrm{hr}$ at $69{ }^{\circ} \mathrm{C}$. The polymer was precipitated into water, filtered, dissolved in acetone, reprecipitated in water and dried at $50{ }^{\circ} \mathrm{C}$, resulting in an $83 \%$ yield. The polymer was partially soluble in HMDS at $10 \mathrm{wt} \%$ polymer in the liquid. Following synthesis and purification, poly (TRIS-co-NVP) (10 wt \%) was mixed in HMDS to produce Polymer Formulation 4. The product chemical structure is shown in Figure 3.

\subsection{Polymer Formulation 6}

$3 \mathrm{M}$ Nexcare ${ }^{\mathrm{TM}}$ No Sting Liquid Bandage Spray, a commercially available product, was studied. It is comprised of an acrylate terpolymer, polyphenylmethysiloxane and HMDS. The chemical structure is under confidentiality and can not be disclosed.

\section{7. ${ }^{1}$ H NMR spectroscopy and Molecular weight determination}

Two identical repetitions of polymer formulations were produced in the study. Selected samples from one repetition were sent to be analyzed by Polymer Solutions Incorporated (Blacksburg, VA) for ${ }^{1} \mathrm{H}$ NMR spectroscopy and molecular weight determination.

The $25 \mathrm{mg}$ of each polymer was dissolved in $10 \mathrm{ml}$ deuterated tetrahydrofuran (d4-THF) and transferred to $5 \mathrm{~mm}$ NMR tubes for proton nuclear magnetic resonance $\left({ }^{1} \mathrm{H}\right.$ NMR) spectroscopy analysis, respectively. The solution was analyzed by size exclusion chromatography with multi-angle light scattering (SEC-MALS) to characterize the molecular weight distribution. Light scattering measurements were performed using a miniDAWN TREOS detector from Wyatt Technology Corporation (Santa Barbara, CA). The concentration detector was an Optilab rEX differential refractometer, also from Wyatt. The specific refractive index increment $(\mathrm{dn} / \mathrm{dc})$ values used for the analysis of the polymers were calculated based on a previous assessment.

\subsection{Polymer characterization}

Moisture vapor transmission rate (MVTR) and elongation rate were also examined by standard methods. MVTR was determined by measuring weight loss over time due to the evaporation of water through the polymer film at body temperature $\left(37^{\circ} \mathrm{C}\right)$. Elongation was measured by applying the polymer formulation onto a rubber band, allowing the formulation to dry, and then stretching the rubber band until the polymer film cracked or delaminated. Just prior to this breaking point is the value 


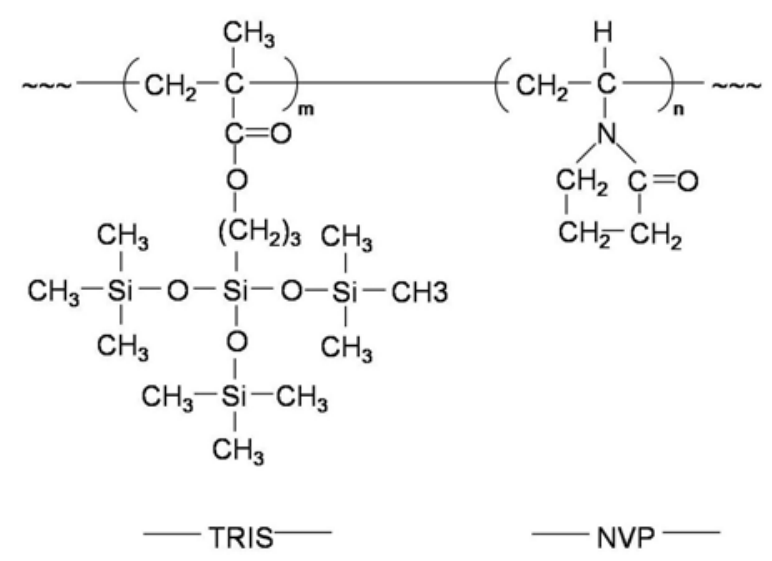

Figure 3. Poly(TRIS-co-NVP) synthesis.

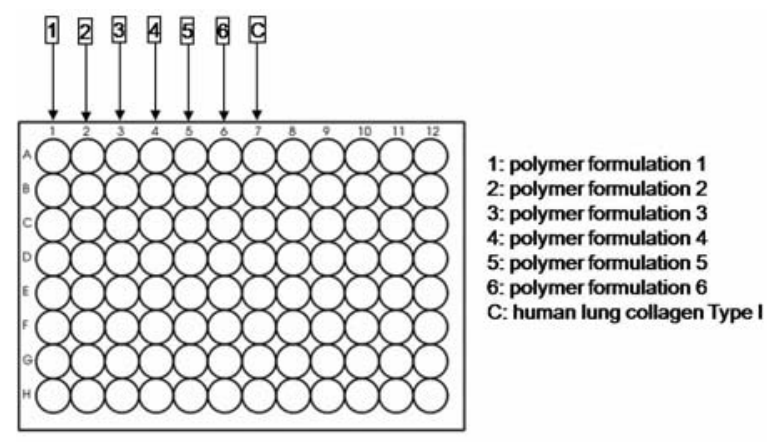

Figure 4. Schematic drawing of Trail III sample plate layout.

for maximum elongation. The surface energy was determined by a set of dyne pens. Polymer thin films were first formed on plastic petri-dishes. Then difference ranges of dyne pens were passed on the polymer films. The surface energy ranges were determined whenever the ink line broke. The contact angle was determined by measuring the angle produced by a drop of DI water on the polymer film surface.

\subsection{Scanning electron microscopy (SEM) imaging}

Polymer samples were dissolved in HMDS to

reach $10 \%(\mathrm{w} / \mathrm{w})$ concentration. $20 \mu \mathrm{l}$ of each polymer formulation was dropped onto metal tabs with diameters of $12 \mathrm{~mm}$ to form thin films for electron microscopy analysis, respectively. Samples were coated with gold, and then examined by JEOL 6335 scanning electron microscope for surface characteristics and porosity of the synthesized copolymers.

\subsection{Cell culture and sample plate preparation}

All the cell culture procedures were carried out under aseptic conditions. HSFs growth medium was made from EMEM supplemented with FBS $(10 \% \mathrm{v} / \mathrm{v})$ and Penicillin/Streptomycin $(1 \% \mathrm{v} / \mathrm{v})$. The serum free HEK growth medium was ready to use after supplementation with antibiotics. Both cells were cultured under the standard cell culture environment in the incubator at $37^{\circ} \mathrm{C}$ with $5 \% \mathrm{CO}_{2}$. Growth media were changed every two days for each cell type. Both cell types reached confluency within a week and were ready to be harvested for further plating.

The 5-day SRB cytotoxicity tests were carried out for HSFs and HEKs on 96-well plates. Polymer formulations 1 through 6 and collagen alone were tested for the SRB assay on columns 1 through 7 while each column had 8 identical replications. $0.01 \mathrm{mg} / \mathrm{ml}$ Human Lung Collagen Type I solution was first coated onto the testing well bottoms to avoid polymer film delamination which was observed in preliminary trials. The plates were then left air-dried inside laminar flow hood in room temperature for about 30 minutes. When the well bottoms were completely dried, different polymer solutions were applied onto the collagen coated well bottoms except for the last column of collagen coating alone. Rapid evaporation of HMDS occurred and yielded clear and adhesive conformal polymer films. Cells with a seeding density of 3400 cells/well were seeded directly onto the polymer films or collagen. Five identical sample plates for the 5-day tests were prepared for each cell type. The schematic drawing in Figure 4 shows the plate layout in detail. Cells on sample plates were incubated under standard culture conditions as previously described. Figure 5 illustrates the schematic procedure for sample plate well preparation for HEKs. Cells were allowed $24 \mathrm{hrs}$ to recover from dissociation trauma before data collection. Thus, Day-1 data was collected after cells were seeded for 48 hrs. Day-2 through Day-5 samples were cultured for an additional $24 \mathrm{hrs}$ in comparison to the previous samples, subsequently.

\subsection{SRB cell viability assay}

All the wells were fixed prior to SRB staining. Taking into account that some fixative might interact with the copolymers, three different fixation methods were evaluated in four different trials. These fixatives were 50 $\%$ trichloroacetic acid (TCA) (Trial I and II), $0.5 \%$ glutaraldehyde (Trial II and III) and $10 \%$ formaldehyde (Trial III and IV). At the end of the fixation period, the liquid in the sample plates was promptly removed by flicking, followed by several washings with distilled water. All samples were kept at room temperature and air dried before SRB staining.

The fixed 96-well plates were stained by $0.1 \%$ SRB for 30 mins at room temperature. The staining solution was then removed and wells were quickly washed 4 times with $1 \%$ acetic acid to remove unbound dye. The washing procedure was also carried out by flicking. The stained sample plates were air dried overnight until no standing moisture was visible. After drying, SRB was solubilized by a $10 \mathrm{mM}$ Trizma base solution and measured at $520 \mathrm{~nm}$ for absorbance data by micro plate reader TECAN. All samples were shaken for 5 mins to facilitate the extraction, solubilization and mixing of SRB dye molecules.

Cell number standard curve analysis for comparing cell numbers was conducted by using 96-well flat bottom plates that were linearly seeded. Cells were 


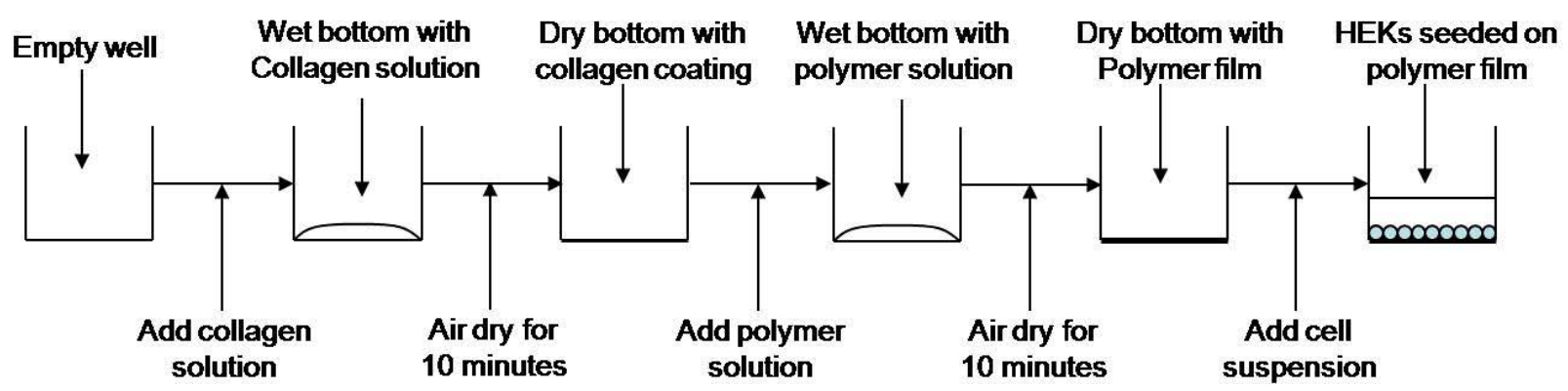

Figure 5. Schematic procedure of HEK sample plate well preparation.

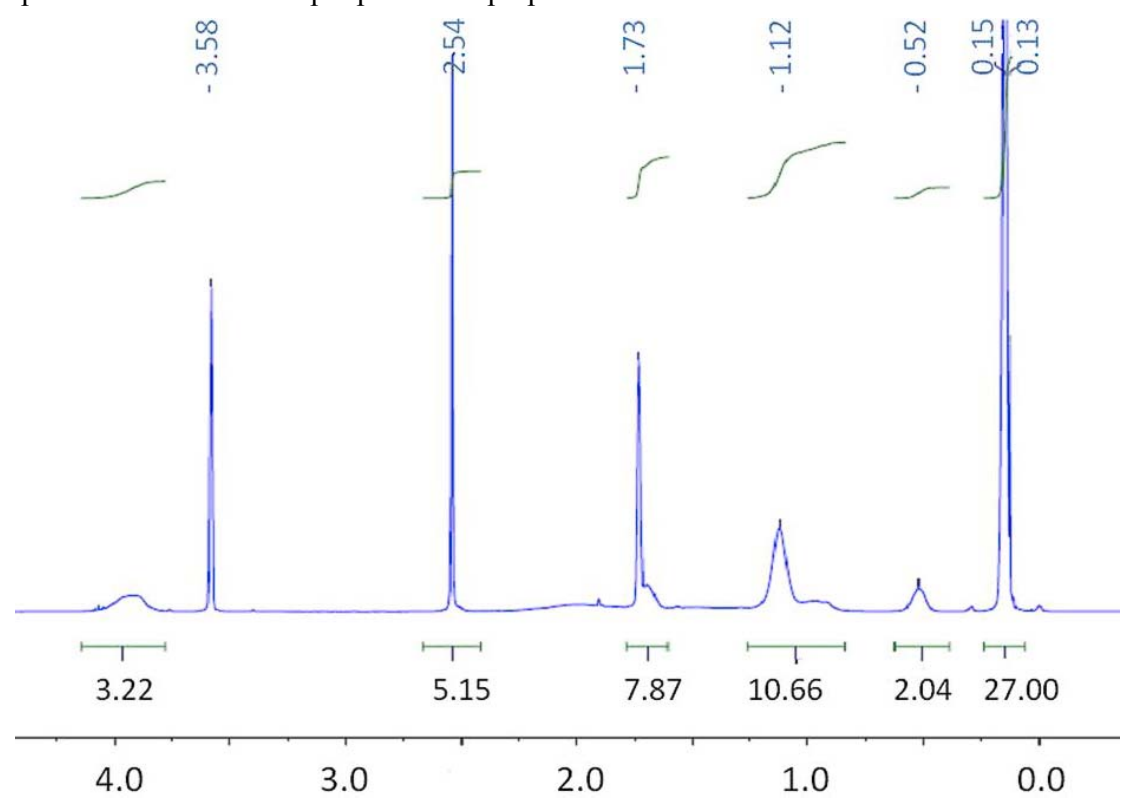

Figure 6. ${ }^{1} \mathrm{H}$ NMR spectrum of poly(TRIS-co-NIPAM).

seeded at the density range of 500 cells/well through 3000 cells/well with a 500 cells/well increment. Standard curve data were collected after $24 \mathrm{hrs}$ to allow recovery from dissociation trauma. Polymer blanks were prepared as correction factors in the SRB assays to be subtracted from the 5 day sample values to normalize absorbance readings. The preparation procedures for polymer blanks were similar to what was previously described except that no cells were seeded onto the polymers. Blank data was also acquired for HMDS (the vehicle) on collagen coated wells.

\subsection{Statistical analysis}

One-way ANOVA statistical analysis produced $\mathrm{P}$ values well below $0.05(\alpha=0.05)$. Significant differences between different copolymers for each of the trials were validated.

\section{RESULTS}

\subsection{Cell viability $S R B$ analysis}

The results of Trial III and Trial IV, both using $10 \%$ formaldehyde as fixative, are reported in this paper. In Trial III, cell viability of HSFs and HEKs were tested on polymer formulations 1 through 6 as substrates including a commercial liquid bandage (Polymer Formulation 6, 3M Nexcare $^{\mathrm{TM}}$ No Sting Liquid Bandage Spray) and on collagen. From the results of Trial III, polymer formulation 2 (poly (TRIS-co-NIPAM) (10 wt \%) with sucrose acetate isobutyrate $(2 \mathrm{wt} \%)$ and $\mathrm{FC}-43)$ and polymer formulation 5 (Poly (TRIS-co-NVP) with FC-43) were found to be not as effective as cell substrates and thus were eliminated in further trials. Thus Trial IV tested cells cultured on polymer formulations 1, 3, 4, 6 and collagen, serving as a replication of Trial III. The results of cell viability on different polymer formulations are shown in Figures 8 through 13 .

\subsection{Polymer characterizations}

According to the cell viability assay results, only the polymer characterization results of polymer formulations 1, 3 and 4 are shown and discussed in this paper. ${ }^{1} \mathrm{H}$ NMR results are shown for only poly (TRIS-coNIPAM). Figure 6 shows the ${ }^{1} \mathrm{H}$ NMR spectrum for the poly (TRIS-co-NIPAM). All of the signals from poly-TRIS are visible, and there is a large broad NIPAM signal observed at $1.12 \mathrm{ppm}$ which corresponds to the isopropyl methyl groups. NIPAM signals near $1.7 \mathrm{ppm}, 2.4 \mathrm{ppm}$, and $3.9 \mathrm{ppm}$ are also expected to be observed, however, these are observed only as the broadening of the TRIS signals 
Table 1. Polymer mechanical characterizations of selected polymer formulations.

\begin{tabular}{|c|c|c|c|c|c|}
\hline Polymer Formulations & $\begin{array}{l}\text { MVRT at } 37^{\circ} \mathrm{C} \\
\left(\mathrm{g} / \mathrm{m}^{2} / 24 \mathrm{hr}\right)\end{array}$ & Elongation Rate & $\begin{array}{l}\text { Surface Energy } \\
\left(\text { dyne/cm }{ }^{2}\right)\end{array}$ & $\begin{array}{l}\text { Contact } \\
\text { Angle }\end{array}$ & Touch \\
\hline $\begin{array}{l}\text { Poly(TRIS-co-NIPAM) } \\
\text { (Polymer Formulation 1) }\end{array}$ & greater than 300 & greater than $40 \%$ & $32-34$ & $50^{\circ}$ & Non-tacky \\
\hline $\begin{array}{l}\text { Poly (TRIS-co-NIPAM-co-DFHMA) } \\
\text { (Polymer Formulation 3) }\end{array}$ & N/A & greater than $80 \%$ & $34-36$ & $55^{\circ}$ & Non-tacky \\
\hline $\begin{array}{l}\text { Poly(TRIS-co-NVP) } \\
\text { (Polymer Formulation 4) }\end{array}$ & $\mathrm{N} / \mathrm{A}$ & greater than $250 \%$ & $36-38$ & $60^{\circ}$ & Non-tacky \\
\hline $3 \mathrm{M}$ Nexcare ${ }^{\mathrm{TM}}$ No Sting (Polymer Formulation 6) & greater than 200 & $10-20 \%$ & N/A & N/A & Non-tacky \\
\hline
\end{tabular}

with which they overlap. The major NIPAM signal overlaps two TRIS signals, however, it is possible to correct for the overlap using the integral ratios of the polyTRIS spectrum to calculate the NIPAM content. The molar percent for TRIS in the copolymer is $43 \%$ and $57 \%$ for NIPAM. Polymer characteristics such as MVTR, elongation rate, contact angle and feel of touch are summarized in Table 1. The SEM image at magnification $10000 \mathrm{x}$ of poly (TRIS-co-NIPAM) is shown in Figure 7. Pictures were taken for all the other polymer formulations but with no identifiable characteristics, thus the results are not shown in this paper.

\section{DISCUSSION}

\subsection{Polymer characterizations}

${ }^{1} \mathrm{H}$ NMR and molecular weight confirmed the molecular ratio of poly (TRIS-co-NIPAM) synthesis. The SEM image in Figure 7 of poly (TRIS-co-NIPAM) showed the porous pattern of the polymer suitable for cell docking and gas permeation (13). Among the three polymers tested for surface energy, poly (TRIS-co-NIPAM) showed the lowest surface energy in the range of 32-34 dyne $/ \mathrm{cm}^{2}$, which means it has the highest hydrophobicity among the three copolymer candidates. This result suggests that the poly (TRIS-co-NIPAM) would serve as a better topical wound dressing since it won't be easily washed off once applied, and it is capable of providing protection for wound edges (13). The relatively high MVTR enables efficient ventilation of the wound area which provides an optimal healing environment. The contact angle results of all the tested polymer formulations revealed the amphiphilic property of this class of copolymers. It was also observed that the LCST of poly (TRIS-co-NIPAM) (polymer formulation 1) was approximately $21^{\circ} \mathrm{C}$. The result was as expected, that the copolymerization with a hydrophobic monomer lowered the LCST of NIPAM, which makes this monomer an excellent candidate in polymeric form to release water and aggregate (by a reduction in polymer chain dimensions) when applied to the human skin or mucosal tissue. Poly (TRIS-co-NIPAM) has the highest moisture vapor transmission rate among all the tested polymer candidates. All the polymer formulations were non-tacky to touch, which means they would all serve as ideal clean skin topical dressings (13).

\subsection{Fixation method optimization}

Because of the interaction between fixative and polymers, the fixative played a critical roll in our particular assessment. Four trials were performed to test different fixation methods as well as to optimize the copolymers that were tested. Trial I tested cells plated on polymers directly coated onto the culture plate wells without any pre- treatment and used the standard $50 \%$ TCA that came with the SRB assay kit as fixative. Shrinkage and contraction of the polymers were observed after TCA fixing, which suggested the standard $50 \%$ TCA was not a suitable fixative in the presence of the polymers. In order to increase the adhesion of the polymer formulations to the culture plate surface, beginning with Trial II all subsequent trials were conducted with polymer formulations casted onto collagen pre-coated 96-well culture plates. No apparent shrinkage was observed for $0.5 \%$ glutaraldehyde fixed samples in Trial II, however, all the polymers tended to delaminate from the well bottoms after the fixation. Hence, glutaraldehyde was demonstrated not to be a good fixative in our assessment. Results revealed that $10 \%$ formaldehyde was a milder fixative for the polymers as minimal interactions were observed between the fixative and copolymers and, in addition, more consistent results were observed throughout the trials. Thus, $10 \%$ formaldehyde fixed sample plates in Trial III and Trial IV were used as our optimal sample preparation in SRB based cytotoxicity assays.

Unlike the normal well plate surfaces, the polymers of this study tended to retain an extra amount of SRB dye resulting in false high optical absorbance readings which would affect the judgment of cell viability. To minimize error on absorbance readings generated from dye absorbed on or pooled within the polymers, absorbance values were measured for "cell-free" polymers stained with $\mathrm{SRB}$ as polymer blank controls. The viable cell numbers shown later have been normalized by subtracting the absorbance readings from the corresponding polymer blanks. Ideally, this normalization provided the absorbance for only the amount of cells, Abscells=Absoriginal Abspolymer. Error occurred due to cell loss by the 'flicking' technique during the washing procedure after fixing. In addition, due to delamination, some samples held media in the well which caused error in the absorbance readings of the SRB staining. Several samples showed a certain degree of inconsistency between the five duplications due to the "flicking" technique and were repeated. When the data was interpreted, outlier data was not included in the calculations as the outlier data were produced from sample delamination and retention of media in the culture plate well and, hence, was not representative of viable cell adhesion onto the polymers during this study.

\subsection{Cell Viability}

Normalized cell number results in Figure 8 indicated that HSFs had discernable viability on polymer formulations 1, 3, 4, 5, 6 as well as on the collagen. Cells could take up to 3 days to recover from dissociation trauma and to adapt to their new growth environment. It appears as 


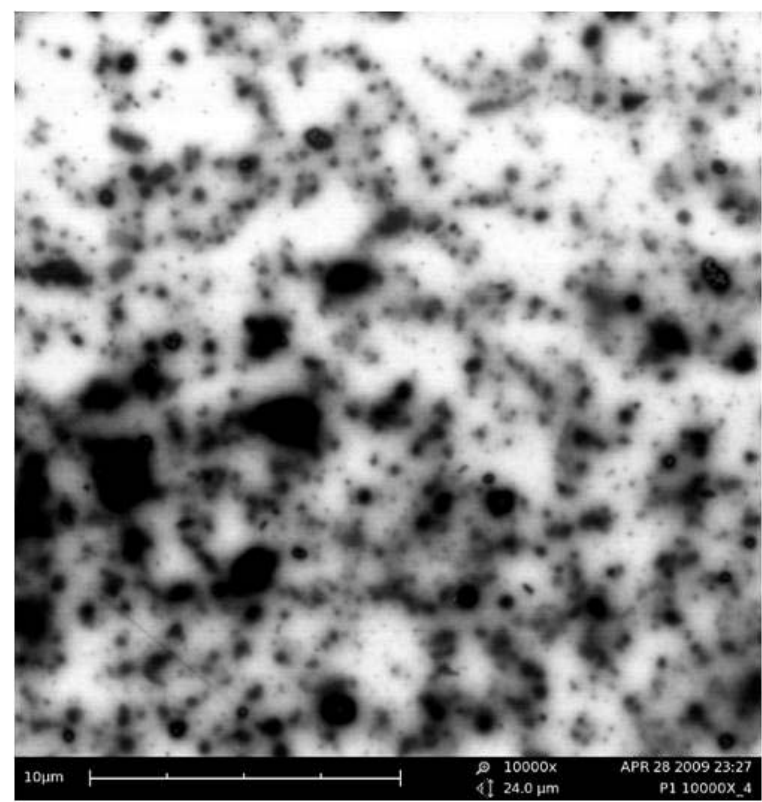

Figure 7. SEM image of poly(TRIS-co-NIPAM) at 10000x magnification.

though the following order of HSF viability was found: polymer formulation $1>4,5$, collagen $>$ polymer formulation $3>$ polymer formulation $6>$ polymer formulation 2. Thus, polymer formulation 1) seemed to provide the best substrate for HSF adhesion as might be expected based on prior research utilizing poly-NIPAM as a cell substrate 2, 6. Poly (TRIS-co-NVP) (polymer formulation 4) and poly (TRIS-co-NVP) with FC-43 (polymer formulation 5) were roughly equivalent to collagen and provided better cell viability than the commercially available polymer formulation 6 , poly (TRIS-co-NIPAM-co-DFHA) with FC-43 (polymer formulation 3), and poly (TRIS-co-NIPAM) with incorporated FC-43 (polymer formulation 2). The incorporation of an oxygen-enriching monomer (DFHA) and the incorporation of FC-43 did not seem to noticeably enhance HSF adhesion. Notably, both polymer formulation 2 and 3, which should have had more incorporated oxygen, seemed to provide the least viable HSF adhesion. Oxygen is reported to have its wound healing effect through tissue perfusion (14). The inconsistent results may be due to in the in vitro environment, cells were seeded onto the polymers, whereas in the actual circumstances it should be the other way around. The fact that oxygen might have equal opportunity to get to the cells virtually in the in vitro setting eliminated the effect of the oxygen-enriching monomers.

Normalized cell number results in Trial IV (Figure 9) indicated that HSFs had better cell viability on poly (TRIS-co-NIPAM) (polymer formulation 1) and poly (TRIS-co-NVP) (polymer formulation 4) than on collagen. Both of the polymer formulations demonstrated show promise as cell substrates for HSFs. Poly (TRIS-coNIPAM-co-DFHA) with FC-43 (polymer formulation 3) and polymer formulation 6 (3M Nexcare ${ }^{\mathrm{TM}}$ No Sting
Liquid Bandage Spray) had negative absorbance (not shown) and negative cell numbers (Figure 9) after normalization indicating lower viable cell adhesion values in comparison to the other polymer formulations and collagen.

In order to observe the effects of the polymer formulations on keratinocytes, another important cell type involved in the wound healing process, same experiments were performed on HEKs as described for HSFs. In Trial III, HEKs grew better on polymer formulations 1, 2, 3, 4 and 6 than on the collagen with the following order of cell viability: polymer formulation $3>1,4>2,6>$ polymer formulation 5 and collagen. Polymer formulation 3 seemed to provide the most HEK adherent viable cells. However, both polymer formulation 1 and 4) behaved similarly and demonstrated cell proliferation on days 4 and 5; standard results for a traditional cell substrate. Polymer formulation 2 and 6 provided less viable cell adhesion than formulations 1 and 4 . Polymer formulation 5 performed the least well in Trial II conditions for HEK cell adhesion and viability. However, it should be noted that when comparing the two cell types, more HEKs survived the processing than HSFs. It is surmised that increased oxygen within the polymer formulation may have improved HEK (which are found in the epidermis) viability as demonstrated by the best viable cell adhesion on polymer formulation 3 . This polymer formulation contained both a perfluorinated monomer within the polymer and a perfluorinated compound known for solubilizing oxygen.

HEKs, as found in Trial IV, again produced higher absorbance readings (not shown) which translated into more cell numbers (Figure 11) as compared to HSFs (Figure 9) using the test methods of this study. Polymer formulation 1 had higher cell viability than collagen or polymer formulation 6 (3M Nexcare ${ }^{\mathrm{TM}}$ No Sting Liquid Bandage Spray) (Figure 11) being roughly equivalent to collagen. Polymer formulation 3 and 4 both had results with low cell counts (varying from positive to negative). Viewing normalized data (Figure 9 and Figure 11), polymer formulation 1 appeared to be a better substrate for HSFs than the other polymer formulations, the commercial polymer formulation or human lung collagen.

Polymer formulation 1 and 4 each demonstrated promise as substrates for HSFs growth based on the reproducibility found between the two trials. These two polymer formulations had noticeably higher cell absorbance readings throughout the five day trials as compared to collagen. Polymer formulation 6 (3M Nexcare ${ }^{\mathrm{TM}}$ No Sting Liquid Bandage Spray) did not appear to support viable HSFs as compared to collagen. Each of the polymers that demonstrated improved cell viability compared to collagen was an amphiphilic nitrogencontaining polymer in contrast to the hydrophobic commercial polymer. The consistent results of improved cell viability and attachment to the amphiphilic nitrogencontaining polymers of this study compared to collagen may be indicative of their usefulness as cell substrates and, potentially, for augmenting wound healing. 


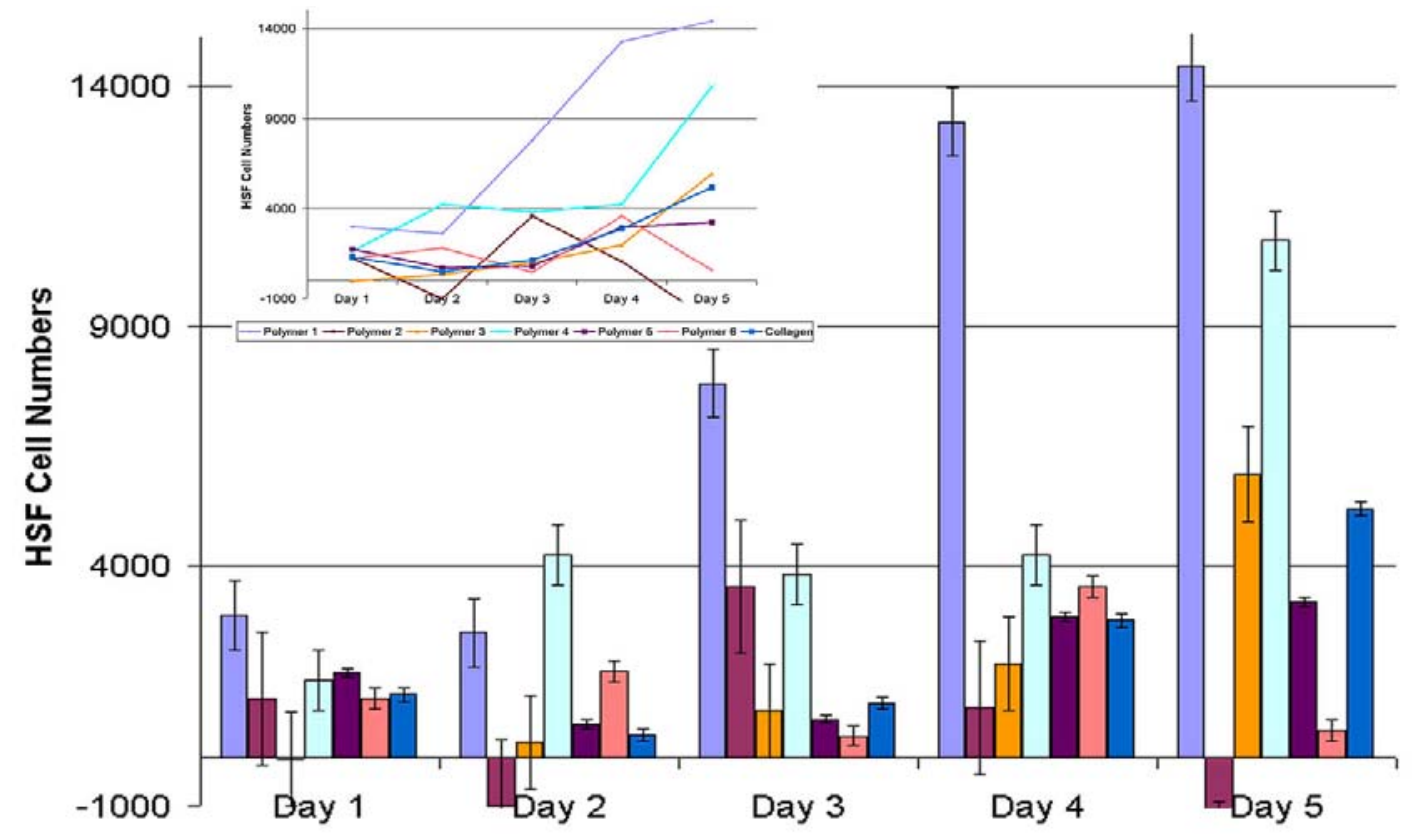

$\square$ Polymer $1 \square$ Polymer $2 \square$ Polymer $3 \square$ Polymer $4 \square$ Polymer $5 \square$ Polymer $6 \square$ Collagen

Figure 8. Normalized cell numbers for HSFs cultured on polymers and collagen in a 5-day period - Trial III.

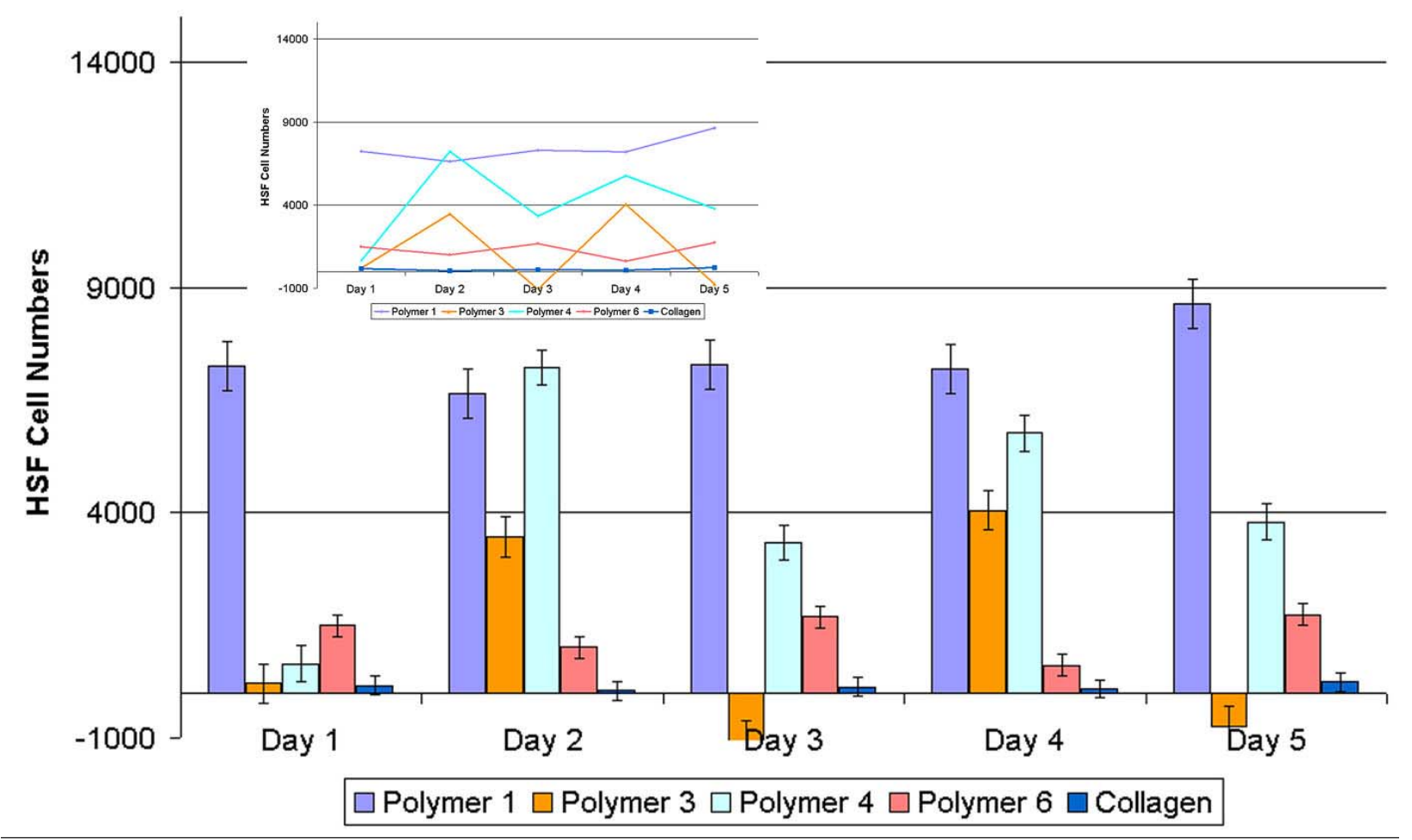

Figure 9. Normalized cell numbers for HSFs cultured on polymers and collagen in a 5-day period - Trial IV. 


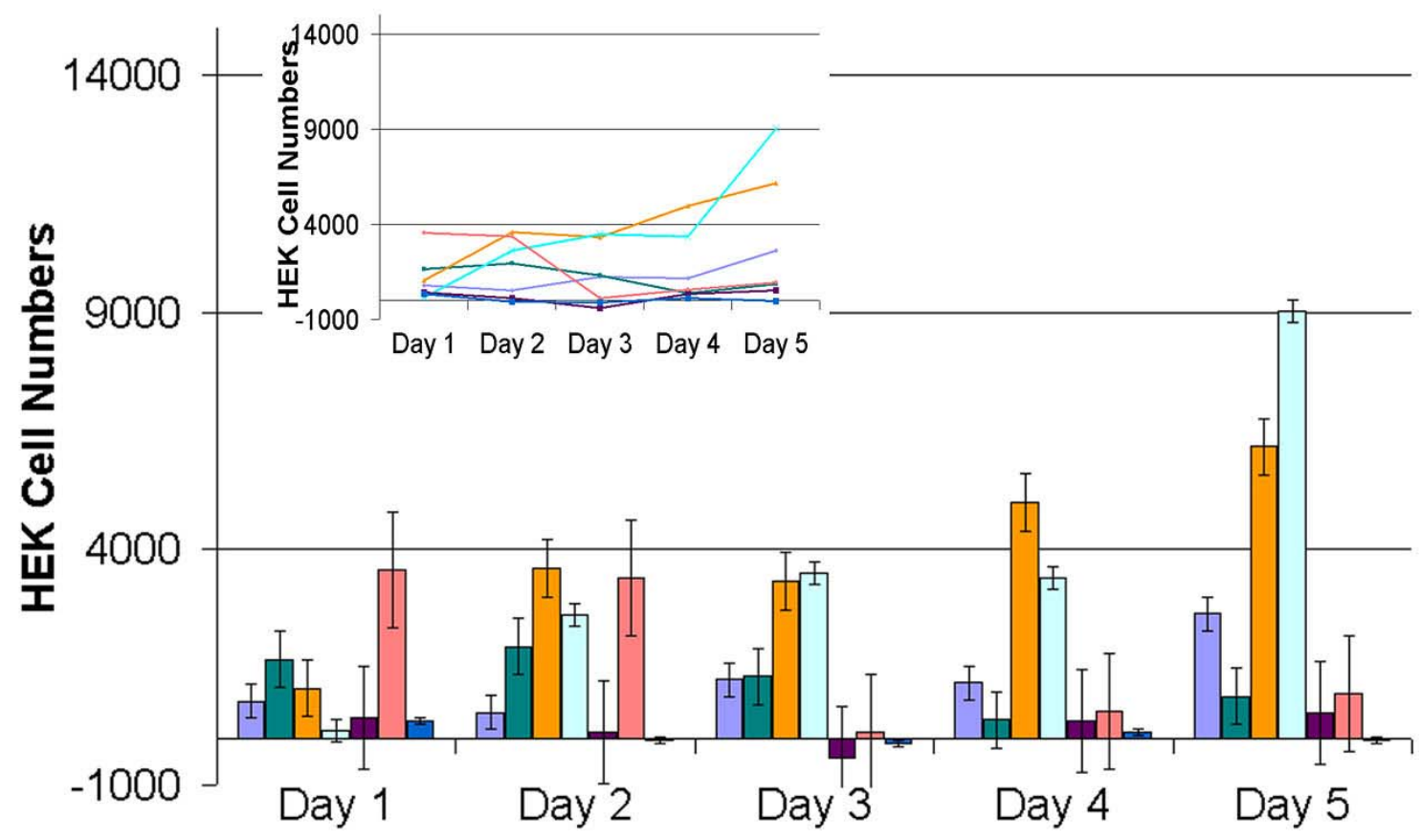

\section{$\square$ Polymer $1 \square$ Polymer $2 \square$ Polymer $3 \square$ Polymer 4 Polymer $5 \square$ Polymer $6 \square$ Collagen}

Figure 10. Normalized cell numbers for HEKs cultured on polymers and collagen in a 5-day period - Trial III.

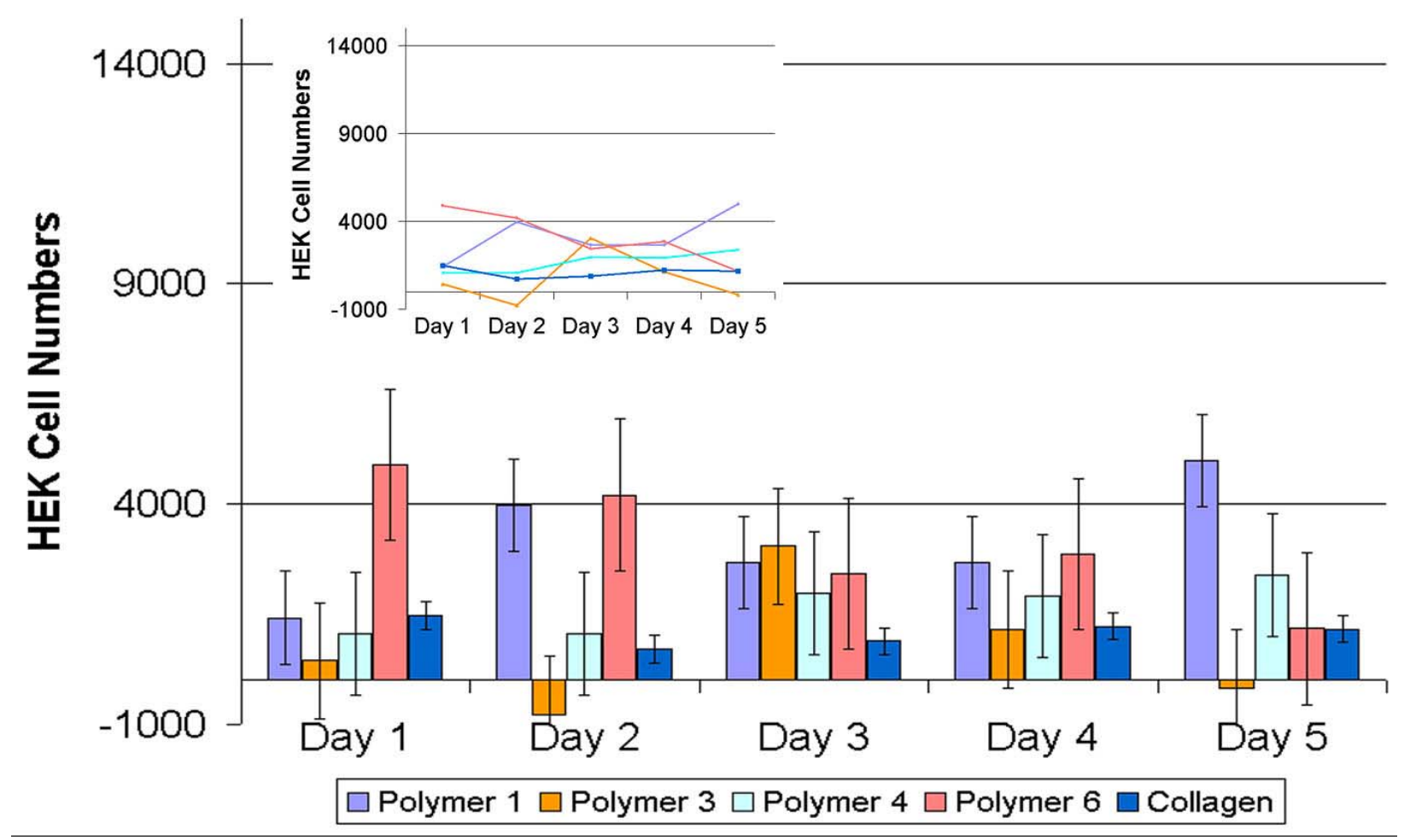

Figure 11. Normalized cell numbers for HEKs cultured on polymers and collagen in a 5-day period - Trial IV. 
Several of the polymers in Trial III demonstrated enhanced HEK cell viability and adhesion as compared to collagen. Poly (TRIS-co-NIPAM-co-DFHA) with FC-43 (polymer formulation 3), which was designed to hold (and then release) more oxygen into the cells, did demonstrate more HEK adhesion on three of the five days of the trial than any of the other polymers. However, this result was not reproduced in Trial IV. During Trial II, the polymers in order of cell viability greater than collagen were: Poly (TRIS-co-NIPAM-co-DFHA) with FC-43 (polymer formulation 3), poly (TRIS-co-NVP) (polymer formulation 4), poly (TRIS-co-NIPAM) with sucrose acetate isobutyrate (polymer formulation 1), poly (TRIS-co-NIPAM) with sucrose acetate isobutyrate and FC-43 (polymer formulation 2), and polymer formulation 6 (3M Nexcare ${ }^{\mathrm{TM}}$ No Sting Liquid Bandage Spray). During Trial IV, which was to be a duplicate of Trial III, only poly (TRIS-co-NIPAM) (polymer formulation 1) performed better than collagen. This lack of consistency may be due to the SRB assay fixation and "flicking" techniques. Also to be noted, in some cases negative normalized absorbance readings were found in both trials which could be expected to have resulted from SRB dye collecting or pooling within the polymer coatings during the washing process and hence creating falsely absorbance.

\section{CONCLUSIONS}

In summary, the new class of liquid bandage copolymers in our study has shown to be promising as the non-cytotoxic substrates in assisting advanced wound healing in terms of promoting cell proliferation. Poly (TRIS-co-NIPAM), the amphiphilic nitrogen-containing copolymer, consistently demonstrated better results of cell viability for both cell types than the other experimental polymers as well as collagen and $3 \mathrm{M}$ NexCare ${ }^{\mathrm{TM}}$ No Sting Liquid Bandage (a hydrophobic polymer). However, each of the amphiphilic nitrogen-containing polymers of this study demonstrated some affinity for viable cell proliferation better than the standard, human lung collagen Type I. These unique polymer coatings of this research could have an impact on advanced wound care.

\section{ACKNOWLEDGEMENT}

This work was supported by Rochal Industries LLP, grant FA9550-07-1-0344 of Department of Defense/ Air Force Office of Scientific Research, and 2008 FIU Faculty Research Award to Dr. Chen-zhong Li.

\section{REFERENCES}

1. Chvapil, M.: Collagen sponge: theory and practice of medical applications. J. Biomed. Mater. Res. 11, 721-741 (1977).

2. Damink, L., P. J. Dijkstra, M. J. A. Van Luyn, P. B. Van Wachem, P. Nieuwenhuis and J. Feijen: Influence of ethylene oxide gas treatment on the in vitro degradation behavior of dermal sheep collagen. J. Biomed. Mater. Res. 29, 149-155 (1995)
3. Bae, Y. H., B. Vernon, C. K. Han and S. W. Kim: Extracellular matrix for a rechargeable cell delivery system. J. Control. Release. 53, 249-258 (1998)

4. Geoffrey, C. G., S. Werner, Y. Barrandon, M. T. Longaker: Wound repair and regeneration. Nature. 453, 314-321 (2008)

5. Kuo, S. M., S. W. Tsai, L. H. Huang and Y. J. Wang: Plasma-modified nylon meshes as supports for cell culturing. Artifl. Cells. Blood. Sub. 25, 551-562 (1997)

6. Stile, R. A., W. R. Burghardt and K. E. Healy: Synthesis and characterization of injectable poly (Nisopropylacrylamide)-based hydrogels that support tissue formation in vitro. Macromolecules. 32, 7370-7379 (1999)

7. Tsuda, Y., A. Kikuchi, M. Yamato, A. Nakao, Y. Sakurai, M. Umezu and T. Okano: The use of patterned dual thermoresponsive surfaces for the collective recovery as co-cultured cell sheets. Biomaterials. 26, 1885-1893 (2005)

8. Ohya, S., S. Kidoaki and T. Matsuda: Poly (Nisopropylacrylamide) (PNIPAM)-grafted gelatin hydrogel surfaces: interrelationship between microscopic structure and mechanical property of surface regions and cell adhesiveness. Biomaterials. 26, 3105-3111 (2005)

9. Salamone, J. C., A. B. Salamone and M. J. Lowe: Conformable bandage and coating material. US. Patent. App. 11/565,453, (2006)

10. Kuran W. Poly (Propylene Oxide). In: Salamone JC, editor. Concise polymeric materials encyclopaedia. Boca Raton: CRC Press; 1999. p 1278-1279.

11. McGrath JA, Eady RAJ, Pope FM. Anatomy and organization of human skin. In: Burns $\mathrm{T}$, Breathnach $\mathrm{S}$, Cox N, Ghriffiths C, editors. Rook's Textbook of Dermatology. Wiley-Blackwell Publishing; 2004. p 3.13.15 .

12. Buffey, J. A., A. G. Messenger, M. Taylor, A. T. T. Ashcroft, G. E. Westgate and S. M. Neil: Extracellular matrix derived from hair and skin fibroblasts stimulates human skin melanocyte tyrosinase activity. Brit. $J$. Dermatol. 131, 836-842 (1994)

13. Sussman, G. Management of the Wound Environment with Dressings and Topical Agents. In: Carrie Sussman, Barbara Bates-Jensen, editor. Wound care: a collaborative practice manual. Lippincott Williams and Wilkins. Baltimore, Maryland (2006)

14. Finn, G., Oxygen in Wound Healing and Infection. World J. Surg. 28, 312-315, (2004)

Abbreviations: LCST: lower critical solution temperature; SRB: Sulforhodamine B; HEK: human epidermal keratinocytes; HSF: human skin fibroblast; TRIS: trimethylsiloxy; NVP: N-vinylpyrrolidone; HMDS: 
hexamethyldisiloxane; NIPAM: N-Isopropylacrylamide; EMEM: eagles minimum essential medium; PBS: phosphate buffered saline; HBSS: Hank's balanced salt solution; FBS: fetal bovine serum; TCA: trichloroacetic acid

Key Words: Wound Healing, Liquid Bandage, Cell Viability, Amphiphilic Polymer, SRB assay

Send correspondence to: Chenzhong $\mathrm{Li}$, Nanobioengineering/Bioelectronics Lab, Department of Biomedical Engineering, Florida International University, 10555, West Flagler Street, Miami, FL 33174, USA. Tel: 305-348-0120, Fax: 1 305-348-6954, E-mail: licz@fiu.edu

http://www.bioscience.org/current/vol3E.htm 